\title{
Shintaido in the elderly: The new way for physical and psychological health
}

\author{
Mattia Roppolo ${ }^{1}$, Anna Mulasso $^{1}$, Daniele Magistro ${ }^{1}$, Antonella Roggero $^{2}$, Patrizio Andreoli ${ }^{1}$, \\ Monica Emma Liubicich ${ }^{1 *}$ \\ ${ }^{1}$ Motor Science Research Center, S.U.I.S.M., University of Torino, Torino, Italy; ${ }^{*}$ Corresponding Author: $\underline{\text { monica.liubicich@unito.it }}$ \\ ${ }^{2}$ Department of Psychology, University of Torino, Torino, Italy
}

Received 17 June 2012; revised 15 July 2012; accepted 23 July 2012

\section{ABSTRACT}

The research aims to investigate the effects of a Shintaido practice in terms of physical and psychological functioning in a group of elderly. Forty seniors, of both gender, with a mean age of $(69 \pm 6)$ years, self-sufficient and without highly invalidating diseases participated in the study. The experimental group (EG) attended the Shintaido physical training of 20 weeks (1 hour per session, twice a week), while the control group (CG) maintained his usual routine. The exercise protocol included specific activities of joint mobility, balance and breathing. At the begin and at the end of intervention were administered to both groups the following validated instruments: 1) One-leg Stance test for the measure of monopodalic static balance; 2) 6-Minutes Walking test for the endurance assessment; 3) Self-Efficacy Perception in Physical Activity (APEF) questionnaire for the selfefficacy evaluation. Data were treated with the not-parametric test for paired and unpaired samples, the Spearman correlation and the linear regression. The results show that: 1 ) the EG improves the endurance in walking and the monopodalic balance as well as his self-efficacy after the Shintaido program; 2) there are strong associations among Shintaido physical activity and physical/psychological variables; 3) there is a mediating effect of walking endurance between the participation to Shintaido training and the self-efficacy. The results suggest that a well structured Shintaido training can help to maintain a good level of physical and psychological functioning in old people.

Keywords: Elderly; Physical Activity; Shintaido; Health; Self-Efficacy

\section{INTRODUCTION}

The increase in elderly population and in the number of sedentary individuals leads us to reflect on the need to identify protective factors for maintaining as long as possible a good level of physical and psychological functioning in old citizens.

Physical activity is one of the major protective factors on the elderly health [1] and the age is not a barrier to practice it. The literature shows that regular physical exercise produces both physical and psychological benefits in elderly [2-5]. Physical activity programs for the elderly seem to have a general positive effect on adaptation of the individual, particularly through the mediation of selfefficacy, increasing the ability to manage tasks of daily living [6]. The elderly seem to prefer training with a multifactorial components $[7,8]$ which not only include strength, balance, joint mobility, walking, but also postural activity, such as Tai Chi [9].

However, the majority of researches focuses on the effects of the Western physical programs, like aerobic training, strength exercises and walking protocols, in the elderly. While just in recent time has placed attention to those of eastern origin [10], among these the Tai Chi and Shintaido exercises. The slowness, the search of relaxation, the balance, the fluidity of movements, the memorization of the sequences and the moderate intensity typical of Tai Chi [11] can produce positive effects both on the physical and psychological functioning of seniors.

In recent years many studies have focused on the effects of Tai Chi in the elderly in terms of hypertension [12], cardiovascular diseases, osteoarthritis [13], cerebrovascular diseases [14] and osteoporosis [15]. Some studies have investigated the effects of Tai Chi on aerobic capacity, falls and balance [11,16,17], muscle strength and postural control [18-20] of old individuals. The studies by Li and colleagues [21-24] showed high levels in the perception of health as well as in self-efficacy related to the ability to fulfill movements [14-24] and, low levels of depression, of negative emotions and of psychological 
distress [21] in elderly Tai Chi practitioners.

There are few studies in literature on the effects of another oriental discipline, similar to Tai Chi, but more recently: the Shintaido. The characteristics of Shintaido are related to the slowness of movement and to the body awareness. The movements are well adapted to the physical characteristics of the elderly because do not take into account the maximal strength and the speed of execution.

The Shintaido is considered an art movement, a "new way of the body" [25]. The activity consists of a series of forms whose purpose is "smooth" and free the body. Each exercise can be designed in a more or less dynamic forms, allowing everyone to work on the edge of their limits. The exercises aim at the opening with segmental or full extension of the body, at the control of breathing, at the maintenance of balance and at the development of safer mobility in the space. The activity begins with the Tenshin-Juso, composed of a series of exercises for flexibility, body awareness and relaxation. Any movement runs from top to bottom of the body, in order to establish a procedure of relaxation appropriate from Heaven to Earth. The initial phase ends with a short meditation followed by a greeting ritual. The practice continues with a series of exercises, "the opening of the body and heart" [26] from the pelvis to the abdomen, chest, hands (palms and fingers), feet, eyes and mouth. The Shintaido needs attention in the alignment of the body segments during movement from the position of the foot to the head, in the sequences and in the organization of the space around the body. The Shintaido, therefore, involves physical and psychological domains: the activity affects aerobic components, muscle strength, joint mobility as well as concentration, attention, visualization, control of energy and mind. In addition, the Shintaido exercises, through reciprocal collaboration in the activities, also influence the social domain.

\subsection{Aims}

The study represents the continuation of a series of research $[27,28]$ that investigate the positive effects of movement on physical functioning and psychological wellbeing in a sample of institutionalized old people. The present study aims to investigate the effects of Shintaido activity in a group of elderly citizens, assuming that the Shintaido, so similar to Tai Chi for movements and purpose, can have positive effects on physical and psychological well-being of participants.

\subsection{Research Questions}

The objectives can be summarized by the following research questions:

1) Can the participation in a Shintaido training program have positive effects on physical (walking endur- ance and balance) and psychological (self-efficacy) variables in a group of elderly? Which are the statistical differences among the elderly who participated in the Shintaido training and those who did not attend it?

2) Which are the associations between the participation to Shintaido training and the change of physical/ psychological variables in elderly? Does exist a relationship between physical and psychological variables?

3) May the walking endurance have a role as a mediator in the direct effect between the participation to the training program and the perception of self-efficacy?

We hypothesize that a Shintaido program improves walking endurance, balance and self-efficacy of elderly and that there are significant statistical differences in physical and psychological variables among participants who attended/not attended to the intervention. More specifically, in the Shintaido group we expect an improvement or maintaining of variables mentioned above while in the not-attended Shintaido program a maintaining or decreasing of the same parameters. Furthermore, we think to observe a positive relationship between the participation to Shintaido training and the change of physical/ psychological aspects as well as between physical and psychological functioning. Finally, we expect to identify a direct effect between the participation to the Shintaido activity and the self-efficacy and, an indirect effect (mediation) of walking endurance on self-efficacy.

\section{METHODS}

\subsection{Procedure}

The research, based on a longitudinal study design, was conducted from January to June 2011 in the province of Verbano-Cusio-Ossola (Piedmont Region, Italy). The first and the last two weeks were dedicated for the baseline (T0) and post-test (T1) assessments. The intervention Shintaido program lasted 20 weeks, from the mid-January to mid-June 2011.

Following the baseline test, participants were randomly included in two groups: the Experimental Group (EG) who took part in the Shintaido exercise program and the Control Group (CG) that maintained their normal routine. Both the groups have carried out the assessments.

\subsection{Participants}

Forty healthy Italian elderly have participated voluntarily in the study. The criteria for participants inclusion were: 1) aged over 60 years; 2) be independent in performing the activities of daily living; 3) living at home; 4) any contraindication to the practice of physical training; 5) absence of highly invalidating chronic diseases (e.g. Alzheimer disease, Parkinson disease, BPCO, heart failure...). The participants accepted the informed consent, according to the Italian law and the ethical code of the 
Italian Association of Psychologists [29].

Participants' age ranged from 60 to 87 years with a mean of $(69 \pm 6)$ years. The $80 \%(\mathrm{~N}=32)$ of participants are female, the $20 \%(\mathrm{~N}=8)$ are male. Most of participants $(75 \%)$ were born in the North, $5 \%$ in the Centre and $10 \%$ in the South of Italy or in the islands; four subjects were born in foreign countries from Italian parents and have moved to Italy within the twenty years. 24 participants (60\%) were married, 13 (about the 32\%) were widowed and 3 were divorced. The level of education corresponded to primary school for the half of participants and to middle and high school diploma respectively for the $28 \%$ and $22 \%$ of the sample. The past job of individuals was been: housewife for $55 \%$, a non-manual work for $21 \%$ (e.g. trader, teacher, secretary), a manual job for the $19 \%$ (e.g. farmer, craftsman, worker) and a women's work for $5 \%$ (e.g. seamstress, domestic) of participants.

With regard to the anthropometric variables, the mean weight of the sample was $(70.0 \pm 13.0) \mathrm{kg}$ with a minimum of $54 \mathrm{~kg}$ and a maximum of $110 \mathrm{~kg}$. The Body Mass Index (BMI) was $(26.5 \pm 5.5) \mathrm{kg} / \mathrm{m}^{2}$ with a range of values from 19.0 to 40.5 . The BMI estimation was obtained by dividing the weight in kilograms of every subject with the square of his height in meters; according to the reference BMI values reported in the clinical guidelines of the National Institutes of Health [30], 20 participants have a normal BMI (from 18.5 to 24.9), 11 were overweight (from 25.0 to 29.9) and 9 were obese (6, 1 e 2 subjects respectively for I, II and III degree of obesity).

The analysis of participants lifestyle showed that only the $5 \%$ of participants smokes and the $10 \%$ consumes regularly alcohol. Furthermore, the $10 \%$ of subjects follows a diet and the same percentage takes drugs for sleeping. Finally, more than $50 \%$ of elderly said to have practiced physical exercise in his life span of whom, the $8 \%$, for resolving a physical problem (e.g. back pain, after surgery, overweight...).

\subsection{Measures}

The balance on a single leg was assessed with the One-Leg Stance test [31] that is one of the most common instrument used in Europe for balance assessment [32]. The operator asks the subject to maintain the balance on a single leg for as long as possible. The stopwatch starts when the not-supported leg rises from the ground and stops when the foot of not-supported leg touches down. The operator must ensure itself that the not-supporting leg is off the ground and not held against the supporting leg, the supporting foot does not move and the arms are kept along the sides. The test is carried out in support of both right and left lower limb, once per leg, and values of the two trials was used for the analysis. The test was consid- ered abnormal when the balance is not maintained for at least 5 seconds [33]. The one-leg stance test is a simple, predictive and inexpensive marker of frailty and insufficient functional mobility in elderly [34,35].

The 6-Minutes Walking test [36] is the dominating European instrument for measure endurance and functional capacity in the elderly [32]. The participants were instructed to walk as quickly as possible for six minutes, along a rectangular course of $(15 \times 7.5)$ m marked with cones. Chairs are positioned along the course for resting. The operator had not provided any kind of encouragement during the test but he communicated at half and 1 minute from the end the time remaining. At the end of the test, participants must stop of walking and the distance walking is recorded. The test was performed in groups of five elderly who were kept constant between pre and post-test. A walking distance range from 400 to 700 meters is considered normal $[37,38]$. The 6-Minutes Walking test is safer and easier to administer than other tests, such as the Shuttle walking test, and reflects the autonomy in activities of daily living of elderly [38,39].

Finally, the self-efficacy was evaluated through the Self-Efficacy Perception in Physical Activity (APEF) [40]. The self-report questionnaire is composed by fourteen items with four answers possibilities, grouped in three scales: 1) intentions to do physical exercise in different conditions (worry, depression, fatigue); 2) to follow the physical activity training program in different conditions (fear of not succeeding, little support, less ability compared to other), 3) to do physical exercises because improves physical conditions, makes you stronger, makes you more confident with your body. The reliability of the scale was investigate in both pre and post test, and the index resulted reliable with a minimum score of Cronbach Alpha of 0.65 and a maximum of 0.95 .

\subsection{Intervention}

The subjects of EG participated in an organized Shintaido physical activity (SPA) of one hour, twice a week in non consecutive days for twenty weeks led by two Shintaido teachers. The training sessions were carried out in a spacious and bright gym easily reached by study participants.

The intervention consisted of three phases of approximately 20 minutes each: 1) Warm up: had included exercises at low intensity, performed in standing/sitting position and in pairs/group using tools, such as sticks and sponge little balls. The exercises were the following: flexibility of the neck, rotation of the shoulders, opening and closing of the hands, circumductions of the pelvis (koshi), flexibility of the spine and flexion and extension of the ankles. Techniques of self-massage to the hands, shoulders, pelvis, abdomen and knees were applied. Also a 
grounding work based on rolling, flexibility and tonification exercises were carried out. 2) Specific physical exercises: were performed: (a) the opening exercises: in tandem stance, antiversion of the pelvis combined with movements of the upper limbs with three level of openness; (b) the Shintaido jump: extension of whole body starting from standing or sitting position with a leap of upper limbs; (c) step exercises from 1 to 4 steps of Shintaido curriculum; (d) Renki "handshake” kumite: in pairs, face each other, shake hands pulling and pushing in all directions, alternating with partner. It is not an exercise for developing strength but for learning to listen to themselves and each other; (e) Tsuki: literally means attack or punch, it is used to express energy; (f) Tenshingoso: is the collection of all the main techniques (attacks, cuts and projections) of Japanese disciplines. It allows to control breathing. Every exercise was also held with the use of voice. 3) Relax and massages: were carried out: (a) Wakame Taisso ("the seaweed and the ocean"), two people in standing position alternately play the role of seaweed and ocean. The seaweed moves itself in relation to the impetus of the ocean; (b) Amma: is the massage in Japan, executed in couples with the hands or foot, are useful for improving well-being of participants.

The beginning and the end of session are dedicated to a brief meditation. Participants had worked on the tasks according to their own skills and they had always been enticed but not forced to do exercises.

\subsection{Statistical Analysis}

Data were analyzed with PASW Statistics-IBM Corporation (USA) through: 1) Descriptive and frequential analysis. 2) The U-Mann Whitney test for unpaired samples. 3) The Wilcoxon test for paired samples with Monte Carlo method calculating the effect size (ES) for the width of relationship. 4) The Spearman correlations analysis with the Rho of Spearman index. 5) The linear regression analysis with the R square coefficient for estimating the goodness of the model. 6) The mediation analysis applying the Barron and Kenny (1986) approach and the Sobel test to estimate the significance and the size of the indirect effect. The regression analysis was employed to test the hypothesis of mediation. The p-value significance was conventionally set at 0.05 .

\section{RESULTS}

\subsection{Baseline Characteristics}

Table 1 shows the baseline characteristics for EG and CG. The two groups are comparable at baseline for the anthropological and demographical (gender and age) variables as well as for the psychological and physical measures. The findings, obtained with U-Mann Whitney test, ensure the baseline homogeneity of EG and CG.
Table 1. Baseline characteristics of participants.

\begin{tabular}{lccc}
\hline VARIABLE & $\begin{array}{c}\text { EG } \\
(\mathrm{n}=20)\end{array}$ & $\begin{array}{c}\text { CG } \\
(\mathrm{n}=20)\end{array}$ & $\mathrm{p}$ \\
\hline $\begin{array}{l}\text { Age, years, } \\
\text { mean } \pm \text { SD }\end{array}$ & $70 \pm 7$ & $69 \pm 5$ & 0.74 \\
$\begin{array}{l}\text { Gender, } \mathrm{n} \\
\begin{array}{l}\text { Female } \\
\text { Male }\end{array}\end{array}$ & 4 & 4 & -- \\
$\begin{array}{l}\text { Weight, kg, mean } \pm \text { SD } \\
\text { Height, cm, mean } \pm \text { SD }\end{array}$ & 16 & 16 & \\
$\begin{array}{l}\text { Body Mass Index, kg/m } \\
\text { mean } \pm \text { SD }\end{array}$ & $160 \pm 8$ & $163 \pm 8$ & 0.17 \\
$\begin{array}{l}\text { One-Leg Stance test Right, sec, } \\
\text { mean } \pm \text { SD }\end{array}$ & $17 \pm 22$ & $18 \pm 17$ & 0.16 \\
$\begin{array}{l}\text { One-Leg Stance test Left, sec, } \\
\text { mean } \pm \text { SD }\end{array}$ & $18 \pm 22$ & $17 \pm 17$ & 0.34 \\
$\begin{array}{l}\text { 6-Minutes Walking test, m, } \\
\text { mean } \pm \text { SD }\end{array}$ & $525 \pm 125$ & $545 \pm 105$ & 0.61 \\
$\begin{array}{l}\text { APEF, questionnaire, } \\
\text { mean } \pm \text { SD }\end{array}$ & $43.5 \pm 5.9$ & $40.4 \pm 3.9$ & 0.16 \\
\hline
\end{tabular}

Note: p based on U-Mann Whitney test for independent sample, SD = standard deviation.

\subsection{Compliance}

All the EG participants completed the 20 weeks training program (compliance 100\%). In particular most of the participants (11/20, 55\%) performed all the training sessions. The rest of participants have the following frequencies: three persons perform 38 sessions, two persons 36 sessions, two persons 34 sessions, one person 30 sessions and 1 person 28 sessions. A percentage of absence lower than $10 \%(48 / 800=6 \%)$ highlights a great compliance of the training protocol.

\subsection{Effects of the Intervention}

The intervention effects in terms of physical functions (walking endurance and balance) and psychological functions (self-efficacy) are shown in Table 2. As mentioned above, in the baseline assessment the EG and the CG were comparable for all the variables, while the introduction of the independent variable (SPA) causes a significant increase in the physical-psychological functions.

\subsubsection{Physical Functions}

The outcomes for the evaluations of physical functions are endurance (6-Minutes Walking test) and balance 
Table 2. The Wicoxon test for dependent sample.

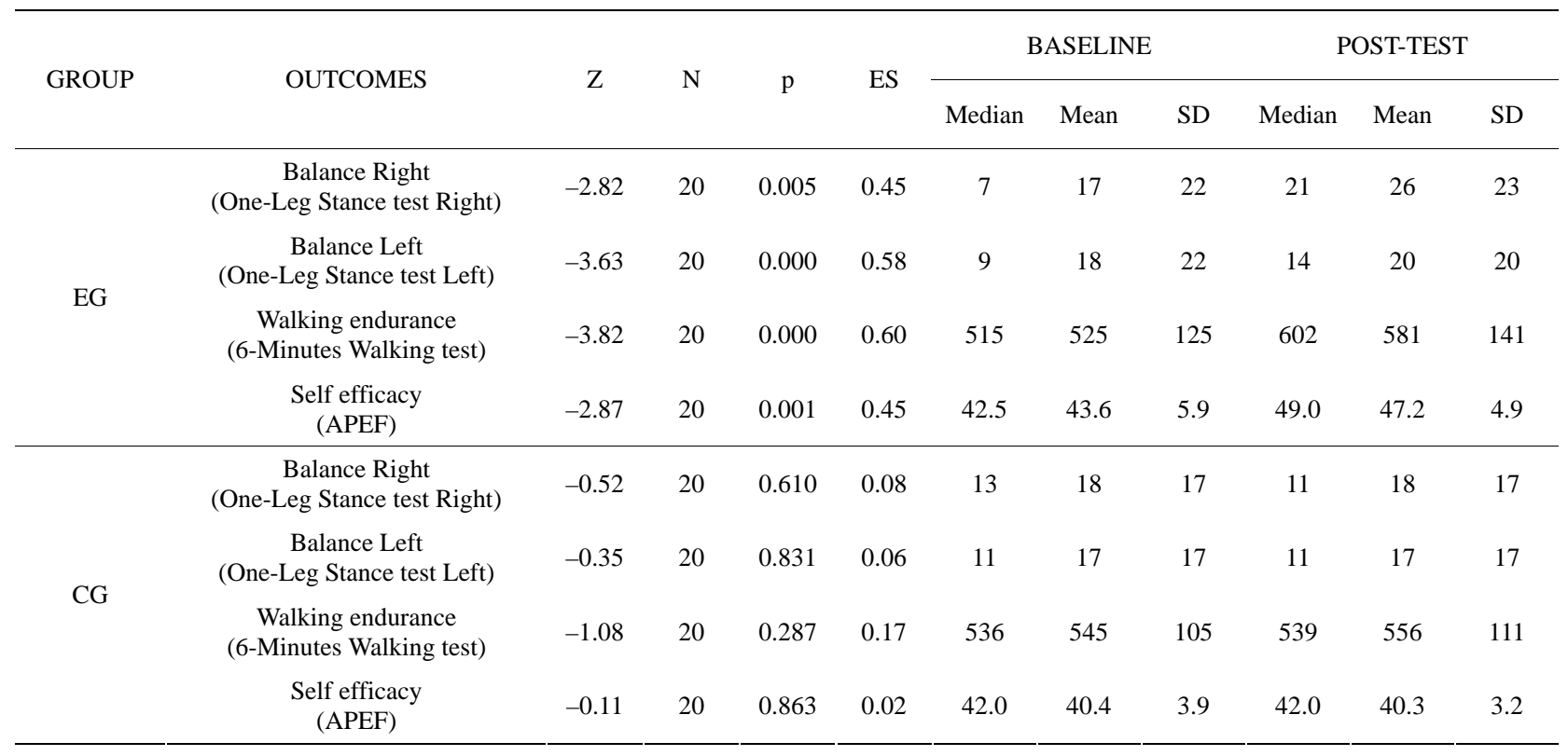

Note: p based on Wilcoxon test for dependent sample, SD = standard deviation, ES = effect size.

(One-Leg Stance test). After the SPA, the EG shows significant differences in both the variables.

1) It increases the level of endurance with an mean performance greater than $56 \mathrm{~m}[\mathrm{~T} 0=(525 \pm 125) \mathrm{m}$, T1 $=(581 \pm 141) \mathrm{m}]$ in the 6-Minutes Walking test $(\mathrm{p}=$ 0.0001 , ES $=0.60$ ). Only a subject doesn't increase the distance in the endurance test, while the other $95 \%$ of participants improves from $2 \mathrm{~m}$ to $173 \mathrm{~m}$. In the same test, the CG doesn't show any significant difference ( $\mathrm{p}=$ 0.287 , $E S=0.17$ ), maintaining its level of endurance.

2) It improves the level of static monopodalic balance (One-Leg Stance test), both on right and left supporting leg. As regards to the trial on the right foot the mean increase has been of $9 \mathrm{~s}[\mathrm{~T} 0=(17 \pm 22) \mathrm{s}, \mathrm{T} 1=(26 \pm 23) \mathrm{s}$, $\mathrm{p}=0.005$, ES $=0.45]$. In the One-Leg Stance test on Right foot six persons have shown a decrease of monopodalic balance, a participant has maintained unchanged its initial level and fourteen subjects have improved their level from a minimum of $1 \mathrm{~s}$ to a maximum of $42 \mathrm{~s}$. Regarding the trial on the Left foot the mean increase has been of $2 \mathrm{~s}$ [T0 $=(18 \pm 22) \mathrm{s}$, T1 $=(20 \pm 20) \mathrm{s}, \mathrm{p}=$ 0.0001 , ES $=0.58]$. No one has decreased its performance in this test, only a subject has maintained his level, while the $95 \%$ of EG has shown a minimum increase of $1 \mathrm{~s}$ and a maximum of $29 \mathrm{~s}$. In the CG there were not statistical differences between T0 e T1 regarding the OneLeg Stance test, both on Right and Left trial (Right foot $\mathrm{p}$ $=0.61, \mathrm{ES}=0.08$; Left foot $\mathrm{p}=0.83, \mathrm{ES}=0.06$ ).

\subsubsection{Psychological Functions}

The introduction of the Shintaido program provides a significant and positive effect in the EG in terms of self- efficacy evaluated through the APEF questionnaire. The mean values of EG are increased from (43.6 \pm 5.9$)$ to (47.2 \pm 4.9$)$, with an associated p value of .001 and an ES of .45. Instead, the CG has maintained its scores without any significant effect $[\mathrm{T} 0=(40.4 \pm 3.9), \mathrm{T} 1=(40.3 \pm$ 3.2), $\mathrm{p}=0.86, \mathrm{ES}=0.02]$.

\subsection{Associations}

The correlations analysis investigates the associations between the SPA and the change (T1-T0) in physical and psychological variables, and if the changes are linked to each others. The results of correlational analysis are reported in Table 3.

There were several significant correlations between the participation in the Shintaido training program and the increase in physical and psychological functions. In particular the SPA is correlated with: 1$)$ self-efficacy $(\mathrm{R}=$ $0.58, \mathrm{p}=0.002) ; 2)$ walking endurance $(\mathrm{R}=0.59, \mathrm{p}<$ 0.0001 ); 3 ) both measures of monopodalic balance (right $\mathrm{R}=0.41, \mathrm{p}=0.008$, Left $\mathrm{R}=0.65, \mathrm{p}=<0.0001$ ). Furthermore, the physical and psychological variables results correlated each others. Self-efficacy is correlated with: 1) walking endurance ( $R=0.39$, $p=0.05)$; 2 ) both measures of monopodalic balance (right $\mathrm{R}=0.50, \mathrm{p}=0.009$; Left $\mathrm{R}=0.39, \mathrm{p}=0.05$ ). Walking endurance is also correlated with the balance on left foot $(\mathrm{R}=0.47, \mathrm{p}=0.002)$ but not with the trial on right.

These results show the great associations between the SPA and the changes in physical and psychological functions as well as the interconnections between walking endurance, balance and self-efficacy. 
Table 3. Correlations.

\begin{tabular}{|c|c|c|c|c|c|c|}
\hline VARIABLES & Index & SPA & $\begin{array}{l}\text { Self-efficacy } \\
\text { change } \\
\text { (APEF) }\end{array}$ & $\begin{array}{l}\text { Balance Right } \\
\text { change } \\
\text { (One-Leg Stance } \\
\text { test Right) }\end{array}$ & $\begin{array}{c}\text { Balance Left } \\
\text { change } \\
\text { (One-Leg Stance } \\
\text { test Left) }\end{array}$ & $\begin{array}{c}\text { Walking endurance } \\
\text { change } \\
\text { (6-Minutes } \\
\text { Walking test) }\end{array}$ \\
\hline \multirow{2}{*}{ SPA } & $\mathrm{R}$ & -- & 0.58 & 0.41 & 0.65 & 0.59 \\
\hline & $\mathrm{p}$ & -- & 0.002 & 0.008 & 0.000 & 0.000 \\
\hline \multirow{2}{*}{$\begin{array}{l}\text { Self efficacy change } \\
\text { (APEF) }\end{array}$} & $\mathrm{R}$ & 0.58 & -- & 0.50 & 0.39 & 0.39 \\
\hline & $\mathrm{p}$ & 0.002 & -- & 0.009 & 0.048 & 0.046 \\
\hline \multirow{2}{*}{$\begin{array}{l}\text { Balance Right change } \\
\text { (One-Leg Stance test Right) }\end{array}$} & $\mathrm{R}$ & 0.41 & 0.50 & -- & 0.51 & 0.10 \\
\hline & $\mathrm{p}$ & 0.008 & 0.009 & -- & 0.001 & 0.535 \\
\hline \multirow{2}{*}{$\begin{array}{l}\text { Balance Left change } \\
\text { (One-Leg Stance test Left) }\end{array}$} & $\mathrm{R}$ & 0.65 & 0.39 & 0.51 & -- & 0.47 \\
\hline & $\mathrm{p}$ & 0.000 & 0.048 & 0.001 & -- & 0.002 \\
\hline \multirow{2}{*}{$\begin{array}{l}\text { Walking endurance change } \\
\text { (6-Minutes Walking test) }\end{array}$} & $\mathrm{R}$ & 0.59 & 0.39 & 0.10 & 0.47 & -- \\
\hline & $\mathrm{p}$ & 0.000 & 0.046 & 0.535 & 0.002 & -- \\
\hline
\end{tabular}

\subsection{Mediation}

SPA-Walking endurance-Self-Efficacy (Table 4). In the first step walking endurance was regressed on SPA. SPA was found to significantly predict endurance level $(\beta$ $=0.58, \mathrm{p}<0.001)$. Then, a hierarchical regression analysis was conducted to examine the successive and independent contributions of (second step) SPA and (third step) of walking endurance on self-efficacy. SPA was a significant predictor of self-efficacy $(\beta=0.43, \mathrm{p}<0.04)$. The effect of SPA was reduced after the walking endurance was entered in the model at step 3. This result was consistent with the presence of a full mediation effect, confirmed by Sobell test $(z=2.60, p<0.01)$.

The final model (see Figure 1) showed that both SPA and endurance were significantly related to self-efficacy. The model as a whole accounted for approximately $40 \%$ of the variance in self-efficacy, with an increase in R2 score from the direct to the whole effect of $25 \%$.

\subsection{Discussion}

This study indicates that a Shintaido training program has influenced both physical and psychological functions of elderly. The analysis carried out allowed the assessment of: 1) the effectiveness of the training, 2) the association between variables and 3) the direct and indirect effects of the participation in a Shintaido program on physical and psychological functions.

Regarding the effects of the intervention, data showed that the EG (that has took part to the Shintaido protocol) has improved the endurance in walking, the balance ability and the self-efficacy, with a medium effect size. In-
Table 4. The mediation model.

\begin{tabular}{|c|c|c|c|}
\hline \multirow{2}{*}{$\begin{array}{l}\text { INDIPENDENT } \\
\text { VARIABLES }\end{array}$} & \multicolumn{3}{|c|}{ DEPENDENT VARIABLES } \\
\hline & First step & Second step & Third step \\
\hline & $\begin{array}{l}\text { Walking endurance } \\
\text { (6-Minutes } \\
\text { Walking test) }\end{array}$ & $\begin{array}{l}\text { Self-efficacy } \\
\text { (APEF) }\end{array}$ & $\begin{array}{l}\text { Self-efficacy } \\
\text { (APEF) }\end{array}$ \\
\hline SPA & $0.58^{* * *}$ & $0.43 *$ & $0.001^{\text {n.s. }}$ \\
\hline $\begin{array}{l}\text { Walking endurance } \\
\text { (6-Minutes } \\
\text { Walking test) }\end{array}$ & -- & -- & $0.67 * *$ \\
\hline F value & 19.00 & 4.9 & 8.8 \\
\hline $\mathrm{R}^{2}$ & 0.32 & 0.15 & 0.40 \\
\hline
\end{tabular}

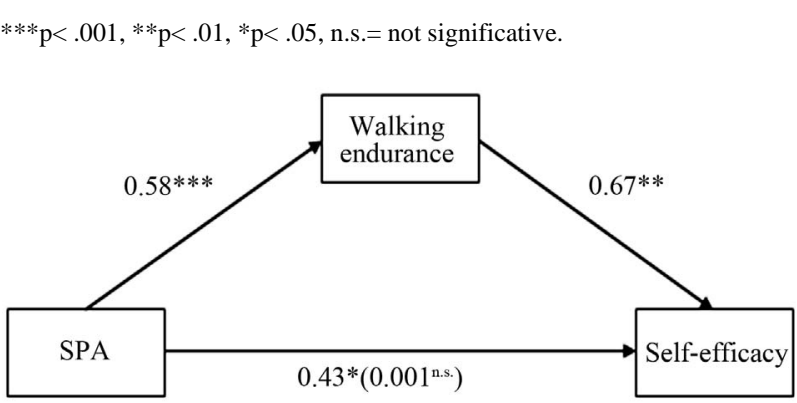

Figure 1. The mediation model. 
stead, the CG showed no statistical differences in the whole battery of tests. These results allow us to answer positively to the first research question.

The findings cited above confirmed the positive effects on both physical and psychological aspects following the involvement in a physical training program [2-5]. In particular some authors [41-43] have found similar results, in terms of endurance, balance and self-efficacy, in a sample of elderly after a physical training. Some researches $[44,45]$ found similar results after the implementation of a Tai Chi training. Our results followed the trends expressed by other research in the same field of interest, but added some innovation aspects: 1) the introduction of a new physical activity protocol, based on an oriental discipline, that combines physical exercises with an activity for the mind; 2) the development of research in an Italian context in which the practice of Shintaido is not yet as common as in the rest of the world.

The association analysis allowed us to understand the strong relationship between the variables under study. In particular with the correlation results we could demonstrate the strong influence of the Shintaido training program on walking endurance, balance and self-efficacy. We found relations between change in psychological and physical functions confirming the importance, especially in the elderly population, of multidisciplinary approach that examine both aspects, as previously described by many authors [46-48]. In summary to the second research question we can answer, increasing the scientific knowledge on elderly health status, that there is strong direct correlation between Shintaido and physical and psychological status, and that these aspects are also positively related to each others.

Finally with the third research question we investigated whether there is a mediating effect of walking endurance between SPA and self-efficacy. As described above some studies $[21,41,43,44]$ highlights the direct effect of different intervention protocols on endurance and self-efficacy. Our study provide also a more complex interpretation of the phenomenon under study, placing the walking endurance in the middle of the direct effect. This results could be very interesting in the constructions of new design studies, that will have to take into account both physical and psychological aspects and test more complex models, and future interventions.

Our study is the first one that tries to investigate the role of Shintaido on physical and psychological health of elderly. The results are very interesting and innovative, but require further confirmation in order to fill up the gaps.

Despite the many strong points, the research presents also some limitations, regarding: 1) the sample size, that is still limited due to the difficulty in involvement of elderly in a oriental discipline that they do not know; 2) the littleness of the geographic area, that makes the sample more homogeneus and complicates the generalizability of results to a broader populations; 3) the gender distribution, that is too much biased towards women; 4) the low number of physical training session, a more prolonged physical training could have provided greater positive effects; 5) the absence of a follow-up test to evaluate the maintenance of physical and psychological benefits over the time.

In conclusion, we think that this study provides the first scientific knowledge on the effects of Shintaido discipline in the elderly population. In the future we hope to enlarge the sample size and try to understand as much as possible the whole pattern of influences of this discipline, also in relation with other types of interventions, in terms of physical and psychological functions.

\section{Conclusions}

Following previous research $[27,28]$, this study tries to understand the way for the maintenance of physical and psychological health in self-sufficient and institutionalized elderly. The present study confirms the effectiveness of the practice of physical activity on both physical and psychological status, underlining the importance to involve elderly in a physical training program. Moreover we demonstrated that the Shintaido discipline can have a strong impact in endurance, balance and self-efficacy, necessary to the maintenance of autonomy in activity of daily living in the elderly population. We think that these results derived from the type of intervention proposed, with slow and controlled movements and a great use of concentration and coordination that can help elderly in the execution of everyday life activities. The performance in Shintaido is always accompanied by meditation, with a complete commitment by the elderly. The combination between physical and psychological effort can explain the effects on both characteristics.

Our results encouraging us to continue the study on the effects of Shintaido training program with the involvement of a larger number of elderly, an increase of training sessions and a comparisons between training protocols (e.g. simple aerobic training and Shintaido). Our final objective is to understand the underlying process to the participation of a physical, psychological or combined intervention in terms of health and autonomy in ability of daily life.

\section{REFERENCES}

[1] Sundquist, K., Qvist, J., Sundquist, J. and Johansson, S.E. (2004) Frequent and occasional physical activity in the elderly: A 12-year follow-up study of mortality. American Journal of Preventive Medicine, 27, 22-27. doi:10.1016/j.amepre.2004.03.011

[2] Baker, M.K., Atlantis, E. and Fiatarone Singh, M.A. 
(2007) Multi-modal exercise programs for older adults. Age Ageing, 36, 375-381.

[3] Netz, Y., Wu, M.J., Becker, B.J. and Tenenbaum, G. (2005) Physical activity and psychological well-being in advanced age: A meta-analysis of intervention studies. Psychol Aging, 20, 272-284.

[4] Patel, K.V., Coppin, A.K., Manini, T.M., Lauretani, F., Bandinelli, S., Ferrucci, L., et al. (2006) Midlife physical activity and mobility in older age: The inchianti study. American Journal of Preventive Medicine, 31, 217-224.

[5] Saxena, S., Van Ommeren, M., Tang, K. and Armstrong, T. (2005) Mental health benefits of physical activity. Journal of Mental Health, 14, 445-451.

[6] McAuley, E., Marquez, D.X., Jerome, G.J., Blissmer, B. and Katula, J. (2002) Physical activity and physique anxiety in older adults: Fitness, and efficacy influences. Aging Ment Health, 6, 222-230. doi:10.1080/13607860220142459

[7] Day, L., Fildes, B., Gordon, I., Fitzharris, M., Flamer, H. and Lord, S. (2002) Randomised factorial trial of falls prevention among older people living in their own homes. British Medical Journal, 325, 128. doi:10.1136/bmj.325.7356.128

[8] Rose, D.J., Jones, J.C. and Lucchese, N. (2002) Promoting functional independence in older adults at risk for falls. Journal of Aging and Physical Activity, 10, 1-9.

[9] Li, F., Harmer, P., Fisher, K.J., McAuley, E., Chaumenton, N., Eckstrom, E., et al. (2005) Tai chi and fall reductions in older adults: A randomized controlled trial. Oxford University Press, Cary.

[10] Frye, B., Scheinthal, S., Kemarskaya, T. and Pruchno, R. (2007) Tai chi and low impact exercise: Effects on the physical functioning and psychological well-being of older people. Journal of Applied Gerontology, 26, 433453. doi:10.1177/0733464807306915

[11] Zhang, J.G., Ishikawa-Takata, K., Yamazaki, H., Morita, T. and Ohta, T. (2006) The effects of tai chi chuan on physiological function and fear of falling in the less robust elderly: An intervention study for preventing falls. Archives of Gerontology and Geriatrics, 42, 107-116.

[12] Lee, M.S., Pittler, M.H., Taylor-Piliae, R.E. and Ernst, E. (2007) Tai chi for cardiovascular disease and its risk factors: A systematic review. Journal of Hypertension, 25, 1974-1975. doi:10.1097/HJH.0b013e32828cc8cd

[13] Lee, M.S., Pittler, M.H. and Ernst, E. (2007) Tai chi for rheumatoid arthritis: Systematic review. Rheumatology, 46, 1648-1651.

[14] Taylor-Piliae, R.E. (2003) Tai chi as an adjunct to cardiac rehabilitation exercise training. Journal of Cardiopulmonary Rehabilitation, 23, 90-96. doi:10.1097/00008483-200303000-00004

[15] Wayne, P.M., Kiel, D.P., Krebs, D.E., Davis, R.B., Savetsky-German, J., Connelly, M., et al. (2007) The effects of tai chi on bone mineral density in postmenopausal women: A systematic review. Archives of Physical Medicine and Rehabilitation, 88, 673-680.

[16] Verhagen, A.P., Immink, M., Van der Meulen, A. and Bierma-Zeinstra, S.M. (2004) The efficacy of tai chi chuan in older adults: A systematic review. Family Practice, 21, 107-113. doi:10.1093/fampra/cmh122

[17] Gatts, S.K. and Woollacott, M.H. (2006) Neural mechanisms underlying balance improvement with short term tai chi training. Aging Clinical and Experimental Research, 18, 7-19.

[18] Maciaszek, J., Osinski, W., Szeklicki, R. and Stemplewski, R. (2007) Effect of tai chi on body balance: Randomized controlled trial in men with osteopenia or osteoporosis. American Journal of Chinese Medicine, 35, 1-9.

[19] Voukelatos, A., Cumming, R.G., Lord, S.R. and Rissel, C. (2007) A randomized, controlled trial of tai chi for the prevention of falls: The central sydney tai chi trial. Journal of the American Geriatrics Society, 55, 11851191.

[20] Yang, Y., Verkuilen, J.V., Rosengren, K.S., Grubisich, S.A., Reed, M.R. and Hsiao-Wecksler, E.T. (2007) Effect of combined taiji and qigong training on balance mechanisms: A randomized controlled trial of older adults. Medical Science Monitor, 13, 339-348.

[21] Li, F., Duncan, T.E., Duncan, S.C., McAuley, E., Chaumeton, N.R. and Harmer, P. (2001) Enhancing the psychological well-being of elderly individuals through tai chi exercise: A latent growth curve analysis. Structural Equation Modeling: A Multidisciplinary Journal, 8, 53-83. doi:10.1207/S15328007SEM0801_4

[22] Li, F., Harmer, P., Chaumeton, N.R., Duncan, T.E. and Duncan, S.C. (2002) Tai chi as a means to enhance selfesteem: A randomized controlled trial. Journal of Applied Gerontology, 21, 70-89. doi:10.1177/073346480202100105

[23] Li, F., McAuley, E., Harmer, P., Duncan, T.E. and Chaumenton, N. (2001) Tai chi enhances self efficacy and exercise behavior in older adults. Journal of Aging and Physical Activity, 9, 161-171.

[24] Li, F., Harmer, P., McAuley, E., Fisher, K.J., Duncan, T.E. and Duncan, S.C. (2001) Tai chi, self-efficacy, and physical function in the elderly. Prevention Science 2, 229-239. doi:10.1023/A:1013614200329

[25] Hokari, S. (1981) Origins: A history of shintaido. Shintaido Publications, Novato.

[26] Quettier, P. (2000) Communication de messages complexes par des séquences gestuelles: Les kata dans les arts martiaux japonais (école shintaïdo). ANRT.

[27] Ciairano, S., Liubicich, M.E. and Rabaglietti, E. (2010) The effects of a physical activity programme on the psychological wellbeing of older people in a residential care facility: An experimental study. Ageing \& Society, 30, 609-626. doi:10.1017/S0144686X09990614

[28] Liubicich, M.E., Magistro, D., Candela, F., Rabaglietti, E. and Ciairano, S. (2012) Physical activity and mobility function in elderly people living in residential care facilities. "Act on aging": A pilot study. Advances in Physical Education, 2, 54-60. doi:10.4236/psych.2012.35054

[29] AIP (1997) Codice etico della ricerca psicologica. http://www.mopi.it/docs/cd/aipcode.pdf

[30] US-NIH (1998) Clinical guidelines on the identification, 
evaluation and treatment of overweight and obesity in adults.

[31] Bohannon, R.W. (1994) One-legged balance test times. Perceptual \& Motor Skills, 78, 801-802.

[32] Frandin, K., Rydwik, E., Bergland, A., Waaler Loland, N. and Forsen, L. (2005) Eunaapa, European network for action on ageing and physical activity. 1-40.

[33] Rolland, Y., Pillard, F., Klapouszczak, A., Reynish, E., Thomas, D., Andrieu, S., et al. (2007) Exercise program for nursing home residents with Alzheimer's disease: A 1-year randomized, controlled trial. Journal of the American Geriatrics Society, 55, 158-165.

[34] Drusini, A.G., Eleazer, G.P., Caiazzo, M., Veronese, E., Carrara, N., Ranzato, C., et al. (2002) One-leg standing balance and functional status in an elderly communitydwelling population in northeast Italy. Aging Clinical and Experimental Research, 14, 42-46.

[35] Vellas, B.J., Rubenstein, L.Z., Ousset, P.J., Faisant, C., Kostek, V., Nourhashemi, F., et al. (1997) One-leg standing balance and functional status in a population of 512 community-living elderly persons. Aging, 9, 95-98.

[36] Butland, R.J., Pang, J., Gross, E.R., Woodcock, A.A. and Geddes, D.M. (1982) Two-, six-, and 12-minute walking tests in respiratory disease. British Medical Journal, 284, 1607-1608. doi:10.1136/bmj.284.6329.1607

[37] Chetta, A., Zanini, A., Pisi, G., Aiello, M., Tzani, P., Neri, M., et al. (2006) Reference values for the 6-min walk test in healthy subjects 20-50 years old. Respiratory Medicine, 100, 1573-1578.

[38] Enright, P.L. (2003) The six-minute walk test. Respiratory Care, 48, 783-785.

[39] Solway, S., Brooks, D., Lacasse, Y. and Thomas, S. (2001) A qualitative systematic overview of the measurement properties of functional walk tests used in the cardiorespiratory domain. Chest, 119, 256-270. doi:10.1378/chest.119.1.256

[40] Caprara, G. (2001) La valutazione dell'autoefficacia. Costrutti e strumenti, Centro Studi Erickson.
[41] Vogel, T., Lepretre, P.M., Brechat, P.H., Lonsdorfer, E., Benetos, A., Kaltenbach, G., et al. (2011) Effects of a short-term personalized intermittent work exercise program (iwep) on maximal cardio-respiratory function and endurance parameters among healthy young and older seniors. Journal of Nutrition Health and Aging, 15, 905911. doi:10.1007/s12603-011-0087-4

[42] Howe, T.E., Rochester, L., Neil, F., Skelton, D.A. and Ballinger, C. (2011) Exercise for improving balance in older people. Cochrane Database of Systematic Reviews, CD004963. doi:10.1002/14651858.CD004963.pub3

[43] McAuley, E., Katula, J., Mihalko, S.L., Blissmer, B., Duncan, T.E., Pena, M., et al. (1999) Mode of physical activity and self-efficacy in older adults: A latent growth curve analysis. Journals of Gerontology Series B: Psychological Sciences and Social Sciences, 54, 283-292. doi:10.1093/geronb/54B.5.P283

[44] Caminiti, G., Volterrani, M., Marazzi, G., Cerrito, A., Massaro, R., Arisi, A., et al. (2011) Tai chi enhances the effects of endurance training in the rehabilitation of elderly patients with chronic heart failure. Rehabilitation Research and Practice, 2011, 761958.

[45] Wu, G. (2002) Evaluation of the effectiveness of tai chi for improving balance and preventing falls in the older population-a review. Journal of the American Geriatrics Society, 50, 746-754.

[46] Heidrich, S.M. (1993) The relationship between physical health and psychological well-being in elderly women: A developmental perspective. Research in Nursing \& Health, 16, 123-130. doi:10.1002/nur.4770160207

[47] Ruuskanen, J.M. and Ruoppila, I. (1995) Physical activity and psychological well-being among people aged 65 to 84 years. Age Ageing, 24, 292-296. doi:10.1093/ageing/24.4.292

[48] Cho, J., Martin, P., Margrett, J., Macdonald, M. and Poon, L.W. (2011) The relationship between physical health and psychological well-being among oldest-old adults. Journal of Aging Research, 2011, 605041. 